\title{
Resiliência, capacidade funcional e apoio social de pessoas com sequelas de acidente vascular encefálico
}

\section{Resilience, functional capacity and social support of people with stroke sequelae}

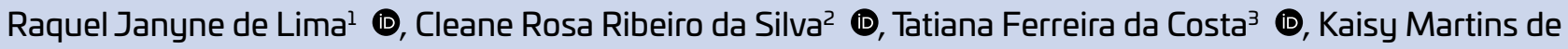 \\ Albuquerque Madruga² (D), Cláudia Jeane Lopes Pimenta² (D), Kátia Neyla de Freitas Macedo Costa² (D)
}

\section{RESUMO}

Objetivo: Investigar a relação entre a resiliência, a capacidade funcional e o apoio social de pessoas com sequelas de acidente vascular encefálico. Método: Estudo transversal, realizado com 108 indivíduos com sequelas de acidente vascular encefálico, cadastrados em Unidades de Saúde da Família, do município de João Pessoa, Paraíba, Brasil. Os instrumentos utilizados foram: Escala de Resiliência, Índice de Barthel e Escala de Apoio Social. Para análise, utilizou-se estatística descritiva e inferencial. Resultados: Houve maior frequência do sexo feminino (57,4\%), 60 anos ou mais $(59,2 \%)$, com companheiro (47,2\%) e cuidador (76,6\%). Os participantes apresentaram mais frequentemente o nível de resiliência moderada (64,8\%), dependência funcional (93,6\%) para realização das atividades diárias e médio apoio social $(48,2 \%)$. Foi verificado que quanto maior a capacidade funcional e o apoio social, maior a resiliência. Conclusáo: A capacidade funcional e o apoio social são preditores da resiliência de pessoas com sequelas de acidente vascular encefálico.

Descritores: Enfermagem; Acidente Vascular Cerebral; Atividades Cotidianas; Apoio Social; Resiliência Psicológica; Reabilitação.

\section{ABSTRACT}

Objective: To investigate the relationship between resilience, functional capacity and social support of people with stroke sequelae. Method: Cross-sectional study, conducted with 108 individuals with sequelae of stroke, registered in Family Health Units, of the municipality of João Pessoa, Paraíba, Brazil. The instruments used were: Resilience Scale, Barthel Index and the Social Support Scale. For analysis, descriptive and inferential statistics were used. Results: There was a higher frequency of females (57.4\%), 60 years old or more $(59.2 \%)$, with partner $(47.2 \%)$ and caregiver $(76.6 \%)$. The participants presented more frequently the level of moderate resilience (64.8\%), functional dependence (93.6\%) for performing daily activities and medium social support (48.2\%). It was verified that the greater the functional capacity and social support, the greater the resilience. Conclusion: Functional capacity and social support are predictors of resilience of people with sequelae of stroke.

Descriptors: Nursing; Stroke; Activities of Daily Living; Social Support; Resilience; Rehabilitation.

\footnotetext{
${ }^{1}$ Auditoria de Contas da Marinha do Brasil - João Pessoa (PB), Brasil. E-mail: raqueljanine@hotmail.com

${ }^{2}$ Universidade Federal da Paraíba - João Pessoa (PB), Brasil. E-mails: cleane rosas@hotmail.com, kaisyjp@hotmail.com, claudinhajeane8@hotmail.com, katianeyla@yahoo.com.br

${ }^{3}$ Universidade Federal de Pernambuco - Vitória de Santo Antão (PE), Brasil. E-mail: tatxianaferreira@hotmail.com
}

Como citar este artigo: Lima RJ, Silva CRR, Costa TF, Madruga KMA, Pimenta CJL, Costa KNFM. Resiliência, capacidade funcional e apoio social de pessoas com sequelas de acidente vascular encefálico. Rev. Eletr. Enferm. [Internet]. 2020 [acesso em: ];22:59542. Disponível em:

https://doi.org/10.5216/ree.v22.59542. 


\section{INTRODUÇÃO}

$\mathrm{O}$ acelerado processo de transição demográfica e epidemiológica tem contribuído para mudança no perfil de adoecimento populacional, gerando o aumento das doenças crônicas. Dados epidemiológicos recentes apontam que entre essas doenças, as cerebrovasculares, especialmente o acidente vascular encefálico (AVE) é considerado, a segunda maior causa de morte no mundo e o Brasil é o país da América Latina que apresenta as maiores taxas de mortalidade ${ }^{(1)}$.

As manifestações clínicas compreendem uma ampla variedade de déficits motores, auditivos, visuais, intelectuais e neurológicos. Somado a isso, estes sujeitos estão expostos a desconfortos físicos, psicológicos e sociais relacionados às sequelas do AVE, ao tratamento e reabilitação ${ }^{(2,3)}$.

Quando o paciente acometido por AVE retorna ao domicílio após alta hospitalar pode se deparar com a dependência de terceiros para realizar as atividades da vida diária (AVDs). A incapacidade funcional retrata diversas mudanças na vida dessas pessoas, como a dificuldade para realizaçáo de atividades de autocuidado, alteração no seu papel familiar, participação social e laboral ${ }^{(4)}$, que pode influenciar na adaptaçáo e na adequação a sua nova condiçáo de vida.

Estudos mostram que a maioria das pessoas acometidas de AVE se torna funcionalmente dependente e experimenta baixa autoestima, isolamento social, ansiedade e depressão, o que repercute negativamente na recuperação, qualidade de vida e sobrevida ${ }^{(4,5)}$. O enfrentamento das adversidades impostas pela doença pode trazer um grande sofrimento, contudo, percebe-se que algumas pessoas conseguem superar as dificuldades e são capazes de utilizar esses acontecimentos como um instrumento para o seu crescimento pessoal ${ }^{(4,6)}$. Esta forma de agir pode estar relacionada com a resiliência.

O termo resiliência é derivado da física, referindo-se às propriedades de um material que retorna à sua forma inicial após ter sofrido uma tensão causadora de deformação elástica ${ }^{(7)}$. Em relação ao ser humano, o conceito de resiliência é um constructo bastante estudado pela Psicologia, sendo definido como uma habilidade de um indivíduo se adaptar de maneira positiva frente às adversidades, traumas, tragédias ou ameaças ${ }^{(8)}$.

A resiliência não é discutida apenas como atributo inato, mas como processo interativo e multifatorial envolvendo aspectos individuais, contexto ambiental e presença dos fatores de proteção e de risco ${ }^{(9)}$. Um dos fatores que pode ser considerado de risco para resiliência prejudicada é a falta de apoio social, que é entendido como um processo dinâmico com intuito de satisfazer as necessidades das pessoas e prover recursos, podendo ter como fontes principais familiares $\mathrm{e}$ profissionais da saúde ${ }^{(7)}$.

A eficácia da rede de apoio é fundamental para a reabilitação do indivíduo que sofreu AVE, uma vez que auxilia na redução de sintomas psicopatológicos e atua na promoçáo da autonomia, independência, capacidade de enfrentamento e adaptação às limitaçôes decorrentes da doença ${ }^{(10)}$. Nesse sentido, evidencia-se que a resiliência do indivíduo sofre influência direta da oferta de uma rede de apoio e suporte para a superação das adversidades ${ }^{(6)}$.

Considerando o aumento da incidência de AVE e da prevalência de incapacidades resultantes desse agravo ${ }^{(5)}$, é importante conhecer os elementos que proporcionem uma melhor condição de vida para essas pessoas, como a resiliência. Esse constructo tem se tornado foco de investigação na produção científica nacional e internacional na área da saúde e da enfermagem ${ }^{(2,9)}$. Todavia, há escassez de estudos que abordam a resiliência nessa população, principalmente relacionada a capacidade funcional e apoio social.

Evidenciar essa relação poderá contribuir para melhoria da qualidade da assistência, a partir da identificação de fatores que influenciam na capacidade de enfrentamento de adversidades de pacientes com sequelas de AVE. Assim, será possível o direcionamento dos profissionais de saúde, entre eles o enfermeiro, na elaboraçấo de um plano de cuidados considerando não apenas os aspectos biológicos, como também os psicossociais, que podem favorecer na recuperação e reabilitação.

Diante do exposto, tem-se como objetivo investigar a relação entre a resiliência, a capacidade funcional e o apoio social de pessoas com sequelas de acidente vascular encefálico.

\section{MÉTODO}

Trata-se de um estudo transversal, realizado com pessoas que possuíam sequelas de AVE, cadastradas em Unidades de Saúde da Família (USF), do município de Joáo Pessoa, PB, Brasil, entre abril e outubro de 2016. A rede de atenção à saúde desse munícipio é composta por 194 USF, administradas de forma organizacional, por meio de cinco Distritos Sanitários. A seleçấo das Unidades foi realizada de forma aleatória, sendo sorteadas 10 de cada Distrito Sanitário, totalizando 50 Unidades.

A população do estudo foi composta por pessoas acometidas por AVE que residiam em João Pessoa/PB. A amostra foi calculada a partir do total de internaçóes hospitalares nos últimos seis meses anteriores à coleta, por meio de dados do Departamento de Informática do Sistema Único de Saúde ${ }^{(11)}$, correspondendo a 149 internaçôes hospitalares.

Os critérios de inclusão foram: possuir idade igual ou superior a 18 anos, ser cadastrado em uma USF e ter pelo menos um tipo de sequela proveniente do AVE, relatada pelo próprio paciente ou cuidador. Decidiu-se investigar adultos e idosos, uma vez que o número de episódios de AVE em jovens vem aumentando ao longo dos anos. Os critérios de exclusão foram: apresentar alguma demência, atraso cognitivo ou doença mental diagnosticada e evidenciar alteraçóes na 
comunicação, na audição ou memória, relatadas por um familiar ou cuidador, uma vez que estas condiçôes poderiam comprometer a coleta dos dados.

O tamanho da amostra foi definido utilizando-se o cálculo para populaçôes finitas com proporçôes conhecidas, tendo-se como base uma margem de erro de $5 \%($ erro $=0,05)$ com grau de confiabilidade de $95 \%\left(\alpha=0,05\right.$, que fornece $\left.Z_{0,05 / 2}=1,96\right)$ e considerando a proporção de participantes de $50 \%(\mathrm{p}=0,5)$, totalizando 108 indivíduos.

Para seleção dos participantes, contataram-se os enfermeiros de cada USF, sendo solicitada a listagem dos pacientes que sofreram AVE e apresentavam sequelas. Destacase que se optou por realizar a entrevista no próprio domicílio do participante, pelo fato da populaçáo de estudo apresentar limitaçóes físicas, dificultando a locomoção.

A coleta de dados de dados foi realizada pelo pesquisador e dois discentes de iniciaçáo científica previamente treinados. Inicialmente, o Agente Comunitário de Saúde (ACS) agendou com o participante o melhor dia e horário para a realizaçáo da visita. No dia marcado, a equipe de pesquisa foi acompanhada do ACS para prestar orientaçóes sobre os objetivos do estudo, solicitar a participação por meio da assinatura ou da impressão dactiloscópica no Termo de Consentimento Livre e Esclarecido e realizar a entrevista. Não houve recusas para a participação no estudo e sete indivíduos foram excluídos.

As entrevistas ocorreram com os 108 pacientes que sofreram alguma sequela de AVE, sendo utilizados quatro instrumentos: dados sociodemográficos (sexo, faixa etária, presença de companheiro, escolaridade e presença de cuidador), Escala de Resiliência, o Índice de Barthel e Escala de Apoio Social. Os dados sociodemográficos utilizados no estudo foram definidos com base em uma busca na literatura a fim de identificar os principais elementos para a caracterização da população com sequelas de AVE.

Utilizou-se a Escala de Resiliência adaptada e validada para o Brasil por Pesce et al. ${ }^{(12)}$, que possui 25 itens, cujas respostas variam de 1 (discordo totalmente) a 7 (concordo totalmente), sendo dividida em três fatores: Resolução de ações e valores (15 itens), Independência e determinaçáo (4 itens) e Autoconfiança e capacidade de adaptação às situaçóes $(6 \text { itens })^{(12)}$.

Os resultados finais variam de 25 a 175 pontos, todavia, em decorrência da escala náo apresentar uma classificaçáo específica para os escores da resiliência, descrevendo apenas que uma pontuação igual ou superior a 147 indicava alta resiliência, optou-se pela distribuição dos valores em forma de quartis. Desta forma, definiu-se Resiliência Baixa os valores de até 111, Resiliência Moderada de 112 a 146 e Resiliência Alta maior ou igual a 147$)^{(12)}$.

O Índice de Barthel foi escolhido por ser abrangente, de fácil aplicação, validado no Brasil ${ }^{(13)}$, e o mais utilizado mundialmente para avaliar a capacidade funcional através das
AVD. Os escores variam de 0 a 20, assim distribuídos: 0 a 4 (dependente muito grave), 5 a 9 (dependente grave), 10 a 14 (dependente moderado), 15 a 19 (dependente leve) e 20 (independente) $)^{(13)}$.

Para avaliação do Apoio Social, foi utilizada a escala do Medical Outcomes Study adaptada para realidade brasileira e validada $^{(14)}$, composta por cinco dimensões: material, afetivo, emocional, informação e interação social, com 19 perguntas em totalidade. Há cinco respostas para cada pergunta: 1 (nunca), 2 (raramente), 3 (às vezes), 4 (quase sempre) e 5 (sempre), os resultados finais oscilam de 19 a 95 pontos $^{(14)}$.

Em virtude de a escala não apresentar classificação específica para o apoio social, neste estudo foram utilizados os valores mínimo e máximo evidenciados pelos participantes (39 e 95 pontos, respectivamente) e distribuídos em forma de quartis. Neste tipo de cálculo, os valores são divididos em três quartis que contemplam quatro partes iguais de $25 \%$, em que o quartil 1 (Q1) corresponde aos $25 \%$ menores valores, o quartil 2 (Q2) delimita os 50\% dos valores e o quartil 3 (Q3) abrange os $25 \%$ maiores valores. Assim, os escores de até 67 pontos correspondem ao apoio social baixo (Q1); entre 68 e 90 pontos, apoio social médio (Q2); e maior ou igual a 91 pontos, apoio social alto (Q3).

Os dados foram armazenados em planilha eletrônica estruturada no Programa Microsoft Excel com dupla digitação, visando garantir a confiabilidade em sua compilação. Posteriormente, foram importados para o software Statistical Package for the Social Sciences (SPSS) versão 22.0 e analisados por meio de estatística descritiva e inferencial, com nível de significância de $5 \%(\mathrm{p} \leq 0,05)$. Considerou-se como variável dependente a resiliência e como independentes a capacidade funcional, o apoio social e as suas dimensóes.

Foi utilizado o teste de Kolmogorov-Smirnov para a verificação da normalidade das variáveis. O teste de correlação de Spearman foi realizado entre a resiliência e as variáveis independentes quantitativas, por apresentarem distribuição não normal. Considerando as variáveis que apresentaram $\mathrm{p} \leq 0,10$ no teste de correlação de Spearman, foi realizada uma análise de regressão linear múltipla para determinar os preditores associados à resiliência. As variáveis com $\mathrm{p} \leq 0,05$ foram consideradas e retidas no modelo final.

A pesquisa foi desenvolvida conforme os aspectos éticos que envolvem seres humanos, preconizados pela Resolução no 466/2012 do Conselho Nacional de Saúde. Houve aprovaçáo pelo Comitê de Ética em Pesquisa do Centro de Ciências da Saúde da Universidade Federal da Paraíba, sob CAAE no 51438115.9 .0000 .5188 e parecer $n^{\circ} 1.418 .615$ de 2016. Os participantes foram devidamente esclarecidos sobre a justificativa da pesquisa, sua finalidade, riscos e benefícios, procedimentos a serem realizados, garantia de sigilo e confidencialidade das informaçóes prestadas e assinaram o Termo de Consentimento Livre e Esclarecido. 


\section{RESULTADOS}

Dos 108 indivíduos participantes, a maioria era do sexo feminino $(57,4 \%)$, com 60 anos ou mais $(59,2 \%)$, casados ou em união estável $(47,2 \%)$, com um a oito anos de estudo $(44,4 \%)$, praticantes da religião católica $(65,7 \%)$, aposentados $(69,2 \%)$, recebiam até R \$ 880 mensais (48,6\%), eram responsáveis pela renda principal da casa $(63,9 \%)$, informaram que a renda não era suficiente para atender as necessidades $(57,9 \%)$, residiam com familiares $(86,1 \%)$ e apresentavam cuidador $(76,6 \%)$, sendo este um membro da família $(91,5 \%)$.

Foi identificada uma maior presença de pessoas que fumavam ou consumiam bebidas alcoólicas (86,1 e 91,5\%, respectivamente), não praticam atividade física $(83,2 \%)$ e não participavam de práticas de lazer $(61,7 \%)$, percebiam a própria saúde como regular (60,2\%), que sofreram o AVE há mais de um ano $(77,8 \%)$, utilizavam algum dispositivo para auxiliar na locomoção $(60,2 \%)$ e tinham como principais sequelas da doença as alteraçóes motoras $(37,2 \%)$ e fraqueza muscular $(36,8 \%)$.

Em relaçáo à avaliação da capacidade funcional, evidenciou-se predomínio de indivíduos com dependência funcional $(93,6 \%)$ grave a muito grave $(20,4 \%)$. Observou-se que a maioria dos pacientes apresentaram apoio social médio $(48,2 \%)$ e baixo $(25,9 \%)$. Os participantes desta pesquisa apresentaram mais frequentemente o nível de resiliência moderada $(64,8 \%)$, seguida de baixa $(28,7 \%)$ e alta $(6,5 \%)$.

A resiliência apresentou correlação positiva e estatisticamente significativa com a capacidade funcional $(\mathrm{r}=0,317 ; \mathrm{p}<0,001)$, o apoio social total $(\mathrm{r}=0,285 ; \mathrm{p}=0,003)$ $\mathrm{e}$ as dimensóes de apoio emocional $(\mathrm{r}=0,347 ; \mathrm{p}=0,001)$ e informação $(r=0,305 ; \mathrm{p}=0,001)$ (Tabela 1).

A análise de regressão linear múltipla mostrou que a capacidade funcional, o apoio social geral e as dimensóes emocional e informação mantiveram associação significativa com o escore total da resiliência. Evidenciou-se que um ponto a mais no escore da capacidade funcional aumenta a resiliência em média $34,3 \%$ e no apoio social aumenta a resiliência em $32,3 \%$. Analisando a resiliência com as dimensóes do apoio social separadamente, identificou-se que um ponto a mais na dimensão emocional aumenta a resiliência em média 32,8\%, e na dimensão informação 36,3\% (Tabela 2).

\section{DISCUSSÃO}

$\mathrm{Na}$ realização das AVDs, observou-se predomínio de pessoas com dependência, o qual pode ser justificado pelo comprometimento causado pelas sequelas do AVE, como hemiplegia, disfagia, paralisação facial, fraqueza muscular, déficits de sensibilidade, alteraçôes visuais, afasia, dispraxia oral

Tabela l. Correlação da resiliência entre a capacidade funcional e o apoio social em pessoas com sequelas de acidente vascular encefálico. João Pessoa, PB, 2016.

\begin{tabular}{l|c|c}
\multirow{2}{*}{} & \multicolumn{2}{|c}{ Resiliência } \\
\cline { 2 - 3 } Capacidade funcional & $\mathbf{r}$ & $\mathbf{p}$-valor \\
\hline Apoio social total & 0,317 & $<0,001$ \\
\hline Dimensão material & 0,285 & 0,003 \\
\hline Dimensão afetivo & 0,006 & 0,095 \\
\hline Dimensão emocional & 0,182 & 0,052 \\
\hline Dimensão informação & 0,347 & 0,001 \\
\hline Dimensão interação social & 0,305 & 0,001 \\
\hline
\end{tabular}

Fonte: dados da pesquisa, 2016.

Tabela 2. Regressão linear múltipla entre a resiliência com a capacidade funcional e apoio social em pessoas com sequelas de acidente vascular encefálico. João Pessoa, PB, 2016.

\begin{tabular}{l|c|c|c|c|c} 
& $\begin{array}{c}\text { Média } \\
(\mathrm{DP})\end{array}$ & Beta & $\begin{array}{c}\text { Erro } \\
\text { padrão }\end{array}$ & IC95\% & p-valor \\
\hline Capacidade funcional & $13,30(5,09)$ & 0,343 & 0,147 & $0,599-1,932$ & 0,000 \\
\hline Apoio social & $77,08(15,64)$ & 0,323 & 0,141 & $0,182-0,592$ & 0,000 \\
\hline Dimensão material & $94,72(9,94)$ & $-0,152$ & 0,003 & $0,224-0,646$ & 0,100 \\
\hline Dimensão afetivo & $81,29(22,11)$ & $-0,086$ & 0,036 & $-12,399-0,978$ & 0,478 \\
\hline Dimensão emocional & $81,71(23,10)$ & 0,328 & 0,186 & $2,379-8,295$ & 0,001 \\
\hline Dimensão informação & $78,56(26,14)$ & 0,363 & 0,207 & $2,641-7,797$ & 0,001 \\
\hline Dimensão interação social & $69,44(28,92)$ & 0,211 & 0,173 & $0,300-3,190$ & 0,593
\end{tabular}

Fonte: dados da pesquisa, 2016.

IC95\%: intervalo de confiança de 95\%. 
e dispraxia de fala, disartria e déficit cognitivo ${ }^{(4)}$. Neste aspecto, aponta-se a importância do enfermeiro no planejamento da alta ainda no ambiente hospitalar e a continuidade dos cuidados no domicílio, adaptando-o à situaçáo de dependência, com a remoção das barreiras arquitetônicas e orientação os cuidados à saúde e reabilitação( ${ }^{(4)}$.

Os participantes apresentaram um nível de apoio social médio a baixo, o que representa uma situação preocupante, visto que o suporte percebido como disponível e satisfatório contribui de maneira significativa para manutenção e integridade física e psicológica do indivíduo ${ }^{(15)}$. A família é a principal fonte de apoio, contudo, frequentemente se observa o despreparo para enfrentar a dependência do paciente e para estabelecer um plano efetivo de intervençóes, o que pode ocasionar a sobrecarga no cuidador e trazer prejuízos aos aspectos físicos, emocionais e sociais ${ }^{(16)}$.

Estudo realizado com sobreviventes de AVE no interior do nordeste brasileiro evidenciou a presença de fragilidades assistenciais no atendimento hospitalar durante as alteraçóes clínicas da doença, o que foi potencializado no retorno ao contexto domiciliar e comunitário, sobretudo pela falta de orientaçóes em saúde e pela ausência de apoio/suporte ${ }^{(17)}$. Diante disso, percebe-se a existência de falhas no sistema de saúde e a fragmentaçáo do cuidado prestado pelos profissionais, com a oferta de uma assistência focada apenas na doença, em vez de uma atenção centrada nas vulnerabilidades do indivíduo e de seus familiares ${ }^{(16,17)}$.

A maioria dos participantes apresentou um nível de resiliência moderado, o que poderia estar relacionado ao fato de serem pessoas idosas, que em detrimento de sua experiência passada, atribui um significado diferente para as adversidades, tentando avaliar positivamente os desafios impostos, mediante a busca e manutenção de atividades prazerosas, adoção de estratégias de enfrentamento eficazes e utilização do suporte social disponível ${ }^{(8)}$.

A resiliência se apresenta como um fator importante para o enfrentamento das dificuldades relacionadas às doenças crônicas e seu tratamento. Nesse sentido, percebe-se que mecanismos formadores da resiliência são fundamentais para o cotidiano dos sobreviventes de AVE, por necessitarem de um cuidado continuado para a reabilitação das sequelas, com realização de fisioterapia, fonoaudiologia, controle nutricional, tratamento medicamentoso, entre outros ${ }^{(3,18)}$, visando minimizar as complicaçóes e evitar a ocorrência de um novo evento.

Com relação à ocorrência de um AVE, muitos são os fatores envolvidos desde o momento do evento até a continuidade dos cuidados no domićlio que podem influenciar na capacidade de enfrentamento do indivíduo acometido, como a funcionalidade e aceitação da família, os recursos financeiros, nível de escolaridade dos envolvidos, crenças espirituais e disponibilidade de serviços de saúde e de informaçôes ${ }^{(4)}$.
Pesquisa realizada com 120 pacientes que apresentavam doenças cardiovasculares no estado de São Paulo evidenciou uma menor resiliência entre os indivíduos com menor autoestima e maiores níveis de depressão e ansiedade ${ }^{(19)}$. Para as pessoas com sequelas de AVE, a baixa resiliência pode interferir na motivação para adesão do tratamento medicamentoso, fisioterapia, mudança nos hábitos alimentares e realizaçáo de atividades de lazer e sociais.

Vale ressaltar, que o termo resiliência foi incorporado na Sistematização da Assistência de Enfermagem, sendo inserido em três diagnósticos da Classificação dos Diagnósticos de Enfermagem da NANDA 2018-2020: Resiliência prejudicada, Risco de resiliência prejudicada e Disposiçáo para resiliência melhorada, os quais fazem parte do Domínio Enfrentamento/Tolerância ao Estresse ${ }^{(20)}$. Portanto, é imprescindível que o enfermeiro identifique esses diagnósticos ao realizar os cuidados ao paciente com sequelas de AVE, pois viabiliza a realização de um planejamento assistencial de acordo com a especificidades de cada um e sua família.

A capacidade funcional foi preditora da resiliência de pessoas com sequelas de AVE, o que demonstra que quanto maior a independência, maior a resiliência, fundamental para manutenção da funcionalidade e do bem-estar subjetivo ${ }^{(8)}$. Corroborando com esta afirmação, estudo realizado na Coreia, no qual foi implementado um programa de exercícios físicos em indivíduos com hemiplegia crônica em decorrência do AVE em hospital de reabilitação, constatou que após oito semanas, os participantes apresentaram, além de melhorias funcionais, maiores níveis de resiliência ${ }^{(21)}$. Assim, entende-se que a capacidade para realizar as atividades diárias repercute no processo de enfrentamento, sendo, portanto, imprescindível as atuaçóes multiprofissionais para proporcionar atividades que favoreçam a independência dessa população.

Evidenciou-se também neste estudo que o apoio social percebido predispôs a resiliência, sobretudo a oferta de suporte na dimensão emocional. Isto demonstra que o fornecimento de suporte após o AVE é fundamental para a reabilitaçáo e consequente aumento da resiliência destes indivíduos, prevenindo doenças mentais que comumente estão associadas, a exemplo da depressão ${ }^{(3)}$.

Em um hospital na China a resiliência e o apoio social estiveram relacionados a um melhor enfrentamento de mulheres com infertilidade ${ }^{(22)}$, assim como em estudo realizado no mesmo país, o qual confirmou que o fornecimento de apoio e maiores níveis de resiliência foram associados à melhoria da qualidade de vida de adultos e idosos com câncer ${ }^{(23)}$.

O apoio social está relacionado ao desenvolvimento da resiliência por meio de mecanismos comportamentais e psicológicos como aumento da autoestima, motivação para adoção de hábitos saudáveis, utilização de estratégias eficazes para o enfrentamento de um evento estressante e o aumento do gerenciamento das emoçôes ${ }^{(24)}$. Além disso, o 
apoio de familiares próximos também favorece a resiliência, uma vez que as relaçóes estabelecidas no ambiente domiciliar atuam como estímulo para a superação da vulnerabilidade e fragilidade diante de sentimentos negativos, fortalecendo a construção da resiliência ${ }^{(22,23)}$.

Salienta-se que esse apoio precisa ser ofertado pelas diversas redes existentes (familiares, amigos, profissionais de saúde e/ ou instituiçóes religiosas), com intuito de melhorar a saúde deste grupo. Dentre as estratégias que favorecem esse fator, pode-se mencionar o aumento da intensidade do contato entre o pacientes e os familiares, amigos e profissionais, avaliação permanente e aconselhamento ${ }^{(16)}$.

Em relação às dimensôes do apoio social, as que tiveram correlação estatisticamente significativa com os níveis de resiliência foi a emocional e de informação. A dimensão de apoio emocional diz respeito a receber demonstraçôes de confiança, empatia, carinho, amor, escuta e interesse, sendo o apoio de informação a disponibilidade de aconselhamentos, sugestôes e informaçóes ${ }^{(14)}$.

Há evidências de que o suporte emocional é fator importante na recuperação da saúde ${ }^{(25)}$, tendo a família papel primordial nesse aspecto, pois a proteção desta e apoio reduzem o isolamento social e contribui para melhor qualidade de vida. Quanto ao apoio de informação, pode ser considerado como mecanismo de participação e cidadania, sendo ofertada a essa população o conhecimento de direitos, auxiliando as estratégias decisórias de escolha e avaliação do tratamento e da reabilitação ${ }^{(15,16)}$.

$\mathrm{O}$ apoio social contribui para o bem-estar, diminuindo a vulnerabilidade diante de situaçóes difíceis ${ }^{(10,15,16)}$ e muitas vezes inesperadas. Assim, é possível perceber a importância do apoio social para o desenvolvimento e/ou fortalecimento da resiliência em pessoas que sofreram AVE, colaborando significativamente com o processo de superaçáo de barreiras e eventos divergentes no decorrer da vida.

É imprescindível o envolvimento de profissionais de saúde, destacando o enfermeiro, em todo o processo de cuidado, desde a internação até o acompanhamento domiciliar, com vista a avaliar o paciente e os fatores de risco para uma resiliência prejudicada $^{(7)}$, elaborando um plano de cuidados que envolva o paciente, a família e a comunidade, no sentido de promover a recuperação, minimizar incapacidades e favorecer a integraçáo social $^{(3,16)}$. Portanto, este estudo tem importantes implicaçóes para prática e pesquisas em enfermagem, pois aponta caminhos para aperfeiçoar a abordagem às pessoas com sequelas de AVE.

As limitaçóes deste estudo referem-se ao delineamento transversal, não sendo possível o acompanhamento, em longo prazo, para avaliar a resiliência, a capacidade funcional e apoio social em cada fase no processo de reabilitação das sequelas da doença. Outra restriçáo é com relação ao instrumento de resiliência utilizado na pesquisa, pois avalia apenas as características individuais, não retratando aspectos ambientais.

\section{CONCLUSÃO}

Os resultados desta pesquisa demostraram que a independência funcional e o apoio social são preditores de maiores médias de resiliência. Ao analisar a resiliência com as dimensóes do apoio social separadamente, observou-se que a resiliência se associou com as dimensóes emocional e informação. Diante da necessidade de estratégias que favoreçam o processo adaptativo inerente às mudanças decorrentes do AVE, o constructo da resiliência surge como uma possibilidade, além de melhorar as condiçôes de vida dessa população.

A repercussão do estudo para a assistência de enfermagem está pautada na relevância da capacidade funcional, do apoio social e da resiliência para o bem-estar e qualidade de vida do sobrevivente de AVE, os quais devem ser investigados durante a internação e monitorados após a alta hospitalar. Neste sentido, torna-se imprescindível a avaliaçáo precoce dos prejuízos funcionais decorrentes das sequelas do AVE, a identificação da rede de apoio disponível, a análise da efetividade do suporte ofertado e a mensuração do nível de resiliência apresentado, a fim de estabelecer um plano de cuidados adequado para a condição de vida e de saúde de cada paciente.

\section{REFERÊNCIAS}

1. Avezum A, Costa-Filho FF, Pieri A, Martins SO, Marin-Neto JA. Stroke in Latin America: burden of disease and opportunities for prevention. Glob Heart [Internet]. 2015 [acesso em: 01 dez. 2018];10(4):32331. Disponível em: https://linkinghub.elsevier.com/ retrieve/pii/S2211-8160(14)00018-0

2. Sadler E, Sarre S, Tinker A, Bhalla A, McKevitt C. Developing a novel peer support intervention to promote resilience after stroke. Health Soc Care Community [Internet]. 2017 [acesso em: 09 dez. 2018];25(5):1590600. Disponível em: https://onlinelibrary.wiley.com/ doi/full/10.1111/hsc. 12336

3. Villain M, Sibon I, Renou P, Poli M, Swendsen J. Very early social support following mild stroke is associated with emotional and behavioral outcomes three months later. Clin Rehabil [Internet]. 2017 [acesso em: $29 \mathrm{dez}$. 2018];31(1):135-41. Disponível em: https://journals. sagepub.com/doi/full/10.1177/0269215515623600?u rl ver $=$ Z39.88-2003\&rfr id=ori:rid:crossref.org\&rfr dat $=$ cr pub $\% 3$ dpubmed

4. Faria ACA, Martins MMFPS, Schoeller SD, Matos LO. Care path of person with stroke: from onset to rehabilitation. Rev Bras Enferm [Internet]. 2017 [acesso em: 02 dez. 2018];70(3):520-8. Disponível em: http://www.scielo.br/pdf/reben/v70n3/0034-7167reben-70-03-0495.pdf 
5. Stein LA, Goldmann E, Zamzam A, Luciano JM, Messé SR, Cucchiara BL et al. Association between anxiety, depression, and post-traumatic stress disorder and outcomes after ischemic stroke. Front Neurol [Internet]. 2018 [acesso em: 09 dez. 2018];9(890):19. Disponível em: https://www.ncbi.nlm.nih.gov/pmc/ articles/PMC6224432/pdf/fneur-09-00890.pdf

6. Böell JEW, Silva DMGV, Hegadooren KM. Sociodemographic factors and health conditions associated with the resilience of people with chronic diseases: a cross sectional study. Rev Latino-Am Enfermagem [Internet]. 2016 [acesso em: 25 abr. 2020];24:e2786. Disponível em: https://www.scielo.br/ pdf/rlae/v24/0104-1169-rlae-24-02786.pdf

7. Araújo CLO, Faro ACM. Estudio sobre la resiliência en ancianas del Valle do Paraíba, São Paulo, Brasil. Enfermería Global [Internet]. 2016 [acesso em: 23 abr. 2020];42:81-98. Disponível em: http://scielo.isciii.es/ pdf/eg/v15n42/clinica4.pdf

8. Fontes AP, Neri AL. Resilience in aging: literature review. Ciênc Saúde Coletiva [Internet]. 2015 [acesso em: 11 jan. 2019];20(5):1475-95. Disponível em: http://www. scielo.br/pdf/csc/v20n5/1413-8123-csc-20-05-01475. pdf

9. Carvalho VD, Teodoro MLM, Borges LO. Escala de Resiliência para Adultos: aplicação entre servidores públicos. Aval Psicol [Internet]. 2014 [acesso em: 02 dez. 2018];13(2):287-95. Disponível em: http://pepsic. bvsalud.org/pdf/avp/v13n2/v13n2a16.pdf

10. Lima RJ, Pimenta CJL, Frazão MCLO, Ferreira GRS, Costa TF, Viana LRC et al. Functional capacity and social support to people affected by cerebrovascular accident. Rev Bras Enferm [Internet]. 2019 [acesso em: 14 abr. 2020];72(4):917-23. Disponível em: https://www.scielo.br/pdf/reben/v72n4/0034-7167reben-72-04-0868.pdf

11. Brasil. Ministério da Saúde. Departamento de Informática do SUS. Base de dados das Informaçóes de Saúde: Morbidade hospitalar do SUS - por local de internação - Brasil [Internet]. 2016 [acesso em: 02 jan. 2019]. Disponível em: http://tabnet.datasus.gov.br/cgi/ deftohtm.exe?sih/cnv/mibr.def

12. Pesce RP, Assis SG, Avanci JQ, Santos NC, Malaquias JV, Carvalhaes R. Cross-cultural adaptation, reliability and validity of the resilience scale. Cad Saúde Pública [Internet]. 2005 [acesso em: 19 jan. 2019];21(2):43648. Disponível em: http://www.scielo.br/pdf/csp/ v21n2/10.pdf

13. Minosso JSM, Amendola F, Alvarenga MRM, Oliveira MAC. Validation of the Barthel Index in elderly patients attended in outpatient clinics, in Brazil. Acta Paul Enferm [Internet]. 2010 [acesso em: 19 jan.
2019];23(2):218-23. Disponível em: http://www. scielo.br/pdf/ape/v23n2/en 11.pdf

14. Griep RH, Chor D, Faerstein E, Werneck GL, Lopes CS. Validade de constructo de escala de apoio social do Medical Outcomes Study adaptada para o português no Estudo Pró-Saúde. Cad Saúde Pública [Internet]. 2005 [acesso em: 21 jan. 2019];21(3):703-14. Disponível em: http://www.scielo.br/pdf/csp/v21n3/04.pdf

15. Elias HC, Marzola TS, Molina NPFM, Assunção LM, Rodrigues LR, Tavares DMS. Relation between Family functionality and the household arrangements of the elderly. Rev Bras Geriatr Gerontol [Internet]. 2018 [acesso em: 11 jan. 2019];21(5):562-9. Disponível em: http://www.scielo.br/pdf/rbgg/v21n5/1809-9823rbgg-21-05-00562.pdf

16. Costa TF, Gomes TM, Viana LRC, Martins KP, MacêdoCosta, KNF. Stroke: patient characteristics and quality of life of caregivers. Rev Bras Enferm [Internet]. 2016 [acesso em: 02 dez. 2018];69(5):933-9. Disponível em: http://www.scielo.br/pdf/reben/v69n5/en 0034-7167reben-69-05-0933.pdf

17. Silva JK, Vila VSC, Ribeiro MFM, Vandenberghe L. Survivors' perspective of life after stroke. Rev Eletr Enf [Internet]. 2016 [acesso em: 08 jul. 2020];18:e1148. Disponível em: https://revistas.ufg.br/fen/article/ view/34620/21190

18. Nishio I, Chujo M. Qualitative analysis of the resilience of adult japanese patients with type 1 diabetes. Acta Med [Internet]. 2016 [acesso em: 27 abr. 2020]; 59(3):196-203. Disponível em: https://www.ncbi.nlm. nih.gov/pmc/articles/PMC5050268/

19. Carvalho IG, Bertolli ES, Paiva L, Rossi LA, Dantas RAS, Pompeo DA. Anxiety, depression, resilience and self-esteem in individuals with cardiovascular diseases. Rev Latino-Am Enfermagem [Internet]. 2016 [acesso em: 02 dez. 2018];24:e2836. Disponível em: http://www.scielo.br/pdf/rlae/v24/0104-1169rlae-24-02836.pdf

20. Herdman TH, Kamitsuru S. Diagnósticos de Enfermagem da NANDA-I: Definições e Classificação 2018-2020 [Internet]. 11. ed. Porto Alegre: Artmed; 2018 [acesso em: 02 jan. 2019].

21. Lee Y-C, Yi E-S, Choi W-H, Lee B-M, Cho S-B, Kim J-Y. A study on the effect of self bedside exercise program on resilience and activities of daily living for patients with hemiplegia. J Exerc Rehabil [Internet]. 2015 [acesso em: 19 jan. 2019];11(1):30-5. Disponível em: https://www.ncbi.nlm.nih.gov/pmc/articles/ PMC4378346/pdf/jer-11-1-30.pdf

22. Yu Y, Peng L, Chen L, Long L, He W, Li M et al. Resilience and social support promote posttraumatic growth of women with infertility: the mediating role of 
positive coping. Psychiatry Res [Internet]. 2014 [acesso em: 27 dez. 2018];215(2):401-5. Disponível em: https://linkinghub.elsevier.com/retrieve/pii/S01651781(13)00683-5

23. Li MY, Yang YL, Liu L, Wang L. Effects of social support, hope and resilience on quality of life among Chinese bladder cancer patients: a cross-sectional study. Health Qual Life Outcomes [Internet]. 2016 [acesso em: 21 dez. 2018];6:14-73. Disponível em: https://hqlo. biomedcentral.com/articles/10.1186/s12955-016-0481-z

24. Southwick SM, L Sippel, Krystal J, Charney D, Mayes L, Pietrzak R. Why are some individuals more resilient than others: the role of social support. Word Psychiatry [Internet]. 2016 [acesso em: $02 \mathrm{dez}$. 2018];15(1):77-9. Disponível em: https://www.ncbi. nlm.nih.gov/pmc/articles/PMC4780285/pdf/WPS15-77.pdf

25. Doussoulin A, Najum J, Saiz JL, Molina F. Impacto de la rehabilitación através de la terapia de restricción inducida modificada en la mejora del apoyo social en usuarios con ataque cerebro vascular. Rev Chil Neuro-Psiquiatr [Internet]. 2016 [acesso em: $12 \mathrm{dez}$. 2018];54(3):187-97. Disponível em: https://scielo. conicyt.cl/pdf/rchnp/v54n3/art03.pdf 